\title{
Separation of Coherent and Incoherent Scattering Contributions in Ellipsometric Light Scattering Experiments on Latex Mixtures
}

\author{
Andreas Erbe, ${ }^{\dagger}$ Klaus Tauer, and Reinhard Sigel*** \\ Max Planck Institute of Colloids and Interfaces, Am Mühlenberg 1, 14476 Potsdam-Golm, Germany
}

\begin{abstract}
Mixtures of poly(styrene) latices were used to vary the sample polydispersity for an investigation of polydispersity effects on ellipsometric light scattering (ELS) data. A procedure for high-accuracy ELS measurements is presented and the experimental distinction of coherent and incoherent scattering contributions is demonstrated. The ellipsometric parameters $\tan \left(\Psi_{\mathrm{Q}}\right)$ and $\Delta$ are solely determined from coherent scattering and represent averaged ensemble properties, independent of the width of the size distribution. The change in polydispersity shows up in the parameter $\tan \left(\Psi_{\mathrm{I}}\right)$, which is affected by incoherent contributions. The average particle size detected by ELS can be estimated from the intensity-weighted size distribution. A radial local birefringence in the particles has been detected and attributed to stress birefringence due to the action of the interface tension on the particles during their growth.
\end{abstract}

\section{Introduction}

The investigation of buried colloidal particle/solvent interfaces presents a particular challenge in the characterization of colloidal systems. Optical characterization methods are of special interest for this task because of their flexibility and their nondestructive nature. Though light scattering has a long history for the characterization of colloids, it is usually not sensitive to contributions from the interface between the particles and the surrounding medium. Recently, we introduced ellipsometric light scattering (ELS) for an investigation of such interfaces. ${ }^{1}$ Similar to reflection ellipsometry, where a thin layer on a planar interface is characterized, ELS gives access to thin layers on dispersed spherical colloidal particles. As first applications, the extent of the ion layer around charge-stabilized colloids was investigated, ${ }^{2}$ and the chain tilt angle in phospholipid vesicles was determined. ${ }^{3}$

The excellent resolution of ELS is based on Mie scattering effects, i.e. details of the scattering polarization which are beyond a description of scattering via Born's approximation. ELS is a filter for such details. ${ }^{1}$

In the previous works, the focus was on the characterization of layers around spherical, monodisperse particles. However, the ELS data also contain information about the total size of particles. For monodisperse spheres, the radius determining the ELS signal is supposed to be the same as extracted from other light scattering methods, namely static and dynamic light scattering. Therefore, mixtures of monodisperse spheres are a well-suited system to study the effect of polydispersity on the results, which will affect the characterization of layers surrounding more complex particles. Since sample polydispersity is almost inevitably present in colloidal samples, this issue is of high importance for measurements and data interpretation.

The way in which polydispersity enters the measurment was discussed theoretically in a recent paper laying the foundations

* Corresponding author. Tel.: +4126 3009134. Fax: +41263009747. E-mail: reinhard.sigel@unifr.ch.

Current address: Max-Planck-Institut für Eisenforschung, Max-PlanckStrasse 1, 40237 Düsseldorf, Germany.

* Current address: Adolphe Merkle Institute, University Fribourg, Chemin du Musée 3, Perolles, 1700 Fribourg, Switzerland.

(1) Erbe, A.; Tauer, K.; Sigel, R. Phys. Rev. E 2006, 73, 031406.

(2) Erbe, A.; Tauer, K.; Sigel, R. Langmuir 2007, 23, 452-459.

(3) Erbe, A.; Sigel, R. Eur. Phys. J. E 2007, 22, 303-309. for this work, ${ }^{4}$ where it was found to differ between ELS and classical scattering methods. Similar differences were found for the effect of the illuminating beam profile on the measurements. For classical scattering methods, polydispersity results in a smearing of the data. Details of the scattering curve are washed out and there is a loss of experimental information compared to scattering data of monodisperse samples. ${ }^{5}$ The effect of polydispersity on ELS is fundamentally different. The discussion in ref 4 introduces suitable quantities for an experimental distinction of coherent and incoherent signals (see below), which are defined in analogy to a similar distinction in contributions to small-angle neutron scattering (SANS). ${ }^{6}$ The coherent part represents the averaged properties of a particle ensemble, while deviations from the average by individual particles give rise to an incoherent scattering contribution. The possibility to extract the coherent part only, i.e., the unsmeared averaged ELS signal, usually allows a reliable interface characterization without information loss, even under nonideal experimental conditions.

The first subject of this paper is to check the equivalence of the radii measured with different light-scattering methods. The second, more general topic is an experimental cross-check of the quantities and concepts introduced in ref 4 . For an investigation of the effect of the size distribution on the coherent and incoherent scattering contributions, samples of controlled polydispersity were prepared by mixing colloidal dispersions consisting of poly(styrene) (PS) particles of known sizes. Measurements are compared to results obtained for the original dispersions.

It is frequently possible to describe ELS data by a model of monodisperse particles. The extracted particle parameters are considered as averaged properties of the particle ensemble under study. Such an "average particle approximation" was briefly discussed in a previous paper on vesicles with birefringent shells. ${ }^{3}$ Due to the local tilt angle of the lipid chains which form the vesicle, such a particle's symmetry is broken. Thus, there is a local orientation of birefringence that varies over the vesicle. Still, a model with radial symmetric uniaxial birefringence oriented normal to the vesicle shell provided a suitable description of the ELS data. The local deviations in individual particles from

(4) Sigel, R.; Erbe, A. Appl. Opt. 2008, 47, 2161-2170.

(5) see, for example: Schärtl, W. Light Scattering from Polymer Solutions and Nanoparticle Dispersion; Springer: Berlin, 2007.

(6) Higgins, J. S.; Benoit, H. C. Polymers and Neutron Scattering; Clarendon Press: Oxford, 1996. 
the average optical sample properties do not affect the coherent signal detected by ELS.

This example underlines the importance of the distinction between coherent and incoherent scattering contributions containing average properties and deviations, respectively, for an understanding of ELS measurements. However, for the samples used in ref 3, it is not possible to evaluate the level of approximation experimentally, since reference particles with properties corresponding to the averaged properties of the ensemble are not available. Homogeneous spheres of controlled polydispersity investigated in this work are the most simple system to check experimentally the effect of deviations from the average particle on ELS results.

The paper is organized as follows: section 2 summarizes the main equations needed here. Both, the setup and a detailed measurement protocol of the ELS experiment are described in section 3. ELS investigations on pure particle fractions and mixtures possessing controlled polydispersity in section 4 are the starting point for a comparison of different averaging formulas with the experimental results. A consistent interpretation of ELS results requires a radial symmetric birefringence of the particles. As the physical origin of this finding, interface tension mediated stress birefringence is discussed.

\section{ELS under Nonideal Conditions}

The analog of the ellipsometric ratio $\rho$ in a scattering measurement reads ${ }^{1}$

$$
\rho=\frac{S_{2}}{S_{1}}=\tan (\Psi) \exp (i \Delta)
$$

Here, $S_{1}$ and $S_{2}$ are the complex scattering amplitudes for light polarized perpendicular ( $V V$ geometry) and parallel ( $H H$ geometry) to the scattering plane, respectively. For particles of spherical geometry as considered here, these linear polarization modes are preserved in the scattering process. The ellipsometric parameters are the modulus $\tan (\Psi)$ of the amplitude ratio and the relative phase shift $\Delta$ of the scattering amplitudes of different polarization. The values of $\tan (\Psi)$ and $\Delta$ depend on the scattering angle $\theta$. The ELS sensitivity is highest around a minimum of $\tan (\Psi)$, found for particles smaller than the light wavelength. In analogy to reflection ellipsometry, the scattering angle $\theta_{\mathrm{B}}$ of the minimum is addressed as Brewster angle. ${ }^{1}$

The simple definition of the ellipsometric parameters via eq 1 becomes ambiguous with polydisperse samples. Therefore, generalized quantities were introduced in ref 4 based on the averages $\left\langle\left|S_{1}\right|^{2}\right\rangle,\left\langle\left|S_{2}\right|^{2}\right\rangle$, and $\left\langle S_{1} * S_{2}\right\rangle$, where $S_{1} *$ is the complex conjugate of $S_{1}$. The averaging $\langle$. $\rangle$ contains several operations: (a) averaging over a particle size distribution, (b) averaging over particle positions in a beam profile, and (c) averaging over different wavelengths for quasi-monochromatic light. Since $\left\langle S_{1} * S_{2}\right\rangle$ is the only complex quantity, the relative phase shift is directly identified as

$$
\Delta=\arg \left(\left\langle S_{1} * S_{2}\right\rangle\right)
$$

For the amplitude ratio, several generalizations were introduced: ${ }^{4}$

$$
\begin{aligned}
& \tan \left(\Psi_{\mathrm{I}}\right)=\sqrt{\frac{\left\langle\left|S_{2}\right|^{2}\right\rangle}{\left\langle\left|S_{1}\right|^{2}\right\rangle}} \\
& \tan \left(\Psi_{\mathrm{A}}\right)=\left|\frac{\left\langle S_{1} * S_{2}\right\rangle}{\left\langle\left|S_{1}\right|^{2}\right\rangle}\right|
\end{aligned}
$$

$$
\tan \left(2 \Psi_{\mathrm{Q}}\right)=\frac{2\left|\left\langle S_{1} * S_{2}\right\rangle\right|}{\left\langle\left|S_{1}\right|^{2}\right\rangle-\left\langle\left|S_{2}\right|^{2}\right\rangle}
$$

While the usage of $\tan \left(2 \Psi_{\mathrm{Q}}\right)$ in eq 5 eases the calculation and gives a simpler formula, the transformation to $\tan \left(\Psi_{\mathrm{Q}}\right)$ is still required for a generalization of $\tan (\Psi)$ in eq 1 . For ideal conditions (monodisperse sample and a plane wave illumination with monochromatic light), the three quantities $\Psi_{\mathrm{I}}, \Psi_{\mathrm{A}}$, and $\Psi_{\mathrm{Q}}$ are identical with $\Psi$ in eq 1 . Under real experimental conditions, the generalized amplitude ratios have a different meaning and different properties. The values of $\left\langle\left|S_{1}\right|^{2}\right\rangle$ and $\left\langle\left|S_{2}\right|^{2}\right\rangle$ are proportional to the scattering intensities $I_{V V}$ and $I_{H H}$ in $V V$ and $H H$ geometry, respectively. Thus, two classical light-scattering measurements with different polarization are sufficient to determine $\tan \left(\Psi_{\mathrm{I}}\right)$. The other two quantities involve the cross term $\left\langle S_{1} * S_{2}\right\rangle$ and can be obtained from ELS measurements. Using polarizer-compensator-sample-analyzer (PCSA) geometry (see section 3.1), the intensity $I$ at the detector for unit incident intensity reads ${ }^{4}$

$$
\begin{aligned}
& 4(k r)^{2} I=\left\langle\left|S_{1}\right|^{2}\right\rangle+\left\langle\left|S_{2}\right|^{2}\right\rangle+ \\
& \cos (2 A)\left[\left\langle\left|S_{2}\right|^{2}\right\rangle-\left\langle\left|S_{1}\right|^{2}\right\rangle\right]+ \\
& 2 \sin (2 A)\left|\left\langle S_{1} * S_{2}\right\rangle\right| \sin (2 P-\Delta)
\end{aligned}
$$

Here, $P$ and $A$ denote the angular positions of polarizer and analyzer, $r$ is the distance from the scattering volume to the detector and $k=2 \pi / \lambda$ is the vacuum wave vector of light with wavelength $\lambda$. Only the last term contains the phase shift $\Delta$. The prefactor of this essential term is directly connected to $\tan \left(\Psi_{\mathrm{A}}\right)$ via eq 4 . Therefore, $\tan \left(\Psi_{\mathrm{A}}\right)$ characterizes the sensitivity of the measurement on $\Delta$. Finally, eq 5 for $\tan \left(2 \Psi_{\mathrm{Q}}\right)$ results from an operational definition mimicking nulling ellipsometry in the ELS measurement process. The nulling angles, i.e., the positions $P_{0}$ and $A_{0}$ of $P$ and $A$ for minimum intensity, are connected to $\Psi_{\mathrm{Q}}$ and $\Delta$ in PCSA geometry (see section 3.1 ) via the equations ${ }^{4,7}$

$$
\begin{gathered}
\Psi_{\mathrm{Q}}=\left|A_{0}\right| \\
\Delta=2 P_{0}+\operatorname{sign}\left(\tan \left(A_{0}\right)\right) \times 90^{\circ}
\end{gathered}
$$

Here, $\operatorname{sign}(x)$ is the signum function, with $\operatorname{sign}(x)=1$ for $x$ $>0, \operatorname{sign}(x)=-1$ for $x<0$, and $\operatorname{sign}(0)=0$. Thus, $\tan \left(\Psi_{\mathrm{Q}}\right)$ derived by minimzing eq 6 represents the "amplitude ratio" determined in a real ELS measurement.

The difference between $\tan \left(\Psi_{\mathrm{I}}\right), \tan \left(\Psi_{\mathrm{A}}\right)$, and $\tan \left(\Psi_{\mathrm{Q}}\right)$ is described by the inequalities ${ }^{4}$

$$
\tan \left(\Psi_{\mathrm{A}}\right) \leq \tan \left(\Psi_{\mathrm{Q}}\right) \leq \tan \left(\Psi_{\mathrm{I}}\right) .
$$

The last inequality does not hold close to a form factor minimum, where $\left\langle\left|S_{1}\right|^{2}\right\rangle\left\langle\left\langle\left|S_{2}\right|^{2}\right\rangle\right.$ and $\tan \left(2 \Psi_{\mathrm{Q}}\right)$ becomes negative (see eq 5). Equation 9 becomes an equality under ideal conditions, and a strict inequality for nonideal conditions. The different behavior of $\tan \left(\Psi_{\mathrm{A}}\right), \tan \left(\Psi_{\mathrm{Q}}\right)$, and $\tan \left(\Psi_{\mathrm{I}}\right)$ can be understood in terms of coherent and incoherent scattering contributions. ${ }^{4}$ The coherent part of the scattering is the intensity, which can be extinguished in a nulling ellipsometry experiment. The intensity of the incoherent part, on the other hand, is independent from the settings of $P$ and $A$, so there is no means to eliminate this contribution with the polarization optics. Since $\tan \left(\Psi_{\mathrm{Q}}\right)$ is defined on the basis of the positions of $P$ and $A$ for minimum intensity, it is determined exclusively by the coherent part. On the other hand, $\tan \left(\Psi_{\mathrm{I}}\right)$ can also be determined from measurements other than ELS and does not distinguish between coherent and incoherent scattering.

(7) Azzam, R. M. A.; Bazhara, N. M. Ellipsometry and Polarized Light; Elsevier: Amsterdam, 1977. 


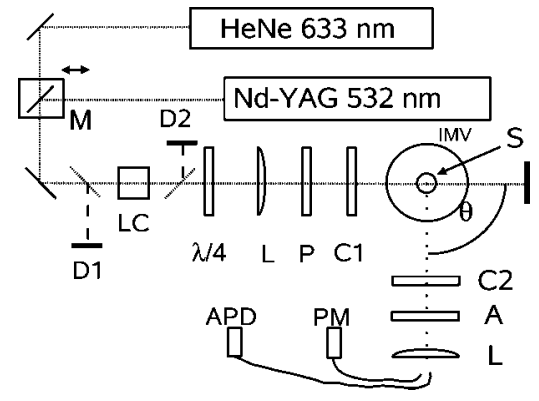

Figure 1. A schematic view of the ELS setup.

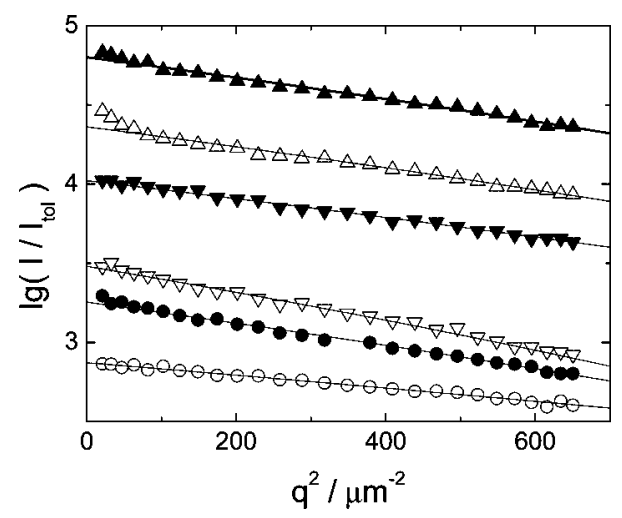

Figure 2. SLS data for the samples (form the upper curve downward) M3, M2, M1, Eur63, Eur62, and Eur41 with fits (lines) of the Mie scattering form factor of a homogeneous sphere. For improved visibility, the data have been offset vertically in steps of 0.35 logarithmic units.

With this background, the goal of this paper is an investigation of the different behavior of $\tan \left(\Psi_{\mathrm{Q}}\right)$ and $\tan \left(\Psi_{\mathrm{I}}\right)$ for a systematic increase of the sample polydispersity, where the incoherent scattering contribution is varied. While a systematic change is expected for the shape of $\tan \left(\Psi_{\mathrm{I}}\right), \tan \left(\Psi_{\mathrm{Q}}\right)$ should remain essentially unchanged, apart from a shift of $\theta_{\mathrm{B}}$ due to the changed average sample size.

\section{Materials and Methods}

3.1. Apparatus. The experimental setup is depicted in Figure 1. A commercial light-scattering goniometer (ALV, Langen, Germany) was equipped with polarization optics (Bernhard Halle, Berlin, Germany), consisting of polarizers ( $\mathrm{P}, \mathrm{A})$ of intensity extinction ratio $10^{-8}$ and compensators $(\mathrm{C} 1, \mathrm{C} 2)$. The magnitude of the birefringence of quartz allows low-order compensators with an effective quarter-wave retardation of opposite sign at the two laser wavelengths $\lambda_{r}=633 \mathrm{~nm}$ (HeNe-Laser PL-3000, Polytec GmbH, Waldbronn, Germany) and $\lambda_{g}=532 \mathrm{~nm}$ (frequency doubled Nd: YAG-Laser DPSS-532-400, Coherent Inc., San Diego, CA). A sliding mirror $(\mathrm{M})$ permits an easy change between the two lasers. The usage of several $\lambda$ adds experimental information and enables the discrimination of effects originating from changes in refractive index from changes in size. Unlike measurements based on Born's approximation, where $\lambda$ and $\theta$ enter commonly through the scattering vector magnitude, ELS relies on Mie scattering, where a change in $\lambda$ cannot be simply compensated by a suitable change in $\theta$. For the experiments reported here, only $\lambda_{\mathrm{r}}$ was available. After monitor photodiodes (D1, D2) and a liquid crystal controller (LC) for variable attenuation (Newport), a lens (L) weakly focuses the light in the sample cell (S), which is placed in an index-matching vat (IMV) filled with toluene. Four stepping-motor-driven rotation stages (Owis, Staufen, Germany) rotate P, C1, C2, and A separately. To achieve full intensity throughput for arbitrary rotation of $\mathrm{P}$, two additional quarter-wave plates are introduced between the lasers and $\mathrm{P}$. The first one $(\lambda / 4)$ transforms the linearly polarized laser light to circular polarization. The second one (not shown in Figure 1) is fixed to $\mathrm{P}$ and rotates with it and, therefore, changes the light's polarization from circular back to linear polarization parallel to the transmission direction of $\mathrm{P}$. In practice, this scheme still contains intensity variations up to $\pm 4 \%$ for different rotations of $P$. A careful calibration of these variations is included as an intensity correction in the data evaluation. On the detection side, the scattered light is coupled by a lens (L) into an optical fiber. The fiber can be connected either to a photomultiplier (PM), which is used for standard experiments as in this work, or to a highly sensitive avalanche photodiode (APD).

In the ELS measurements, $\mathrm{C} 1$ is fixed to the angle of $45^{\circ}$ with respect to the scattering plane, while $\mathrm{C} 2$ is rotated parallel to $\mathrm{A}$ and thereby effectively deactivated (PCSA geometry). ${ }^{7}$

3.2. Nulling Ellipsometry. Compared to the intensity of the reflected beam in a classical reflection ellipsometry experiment, the scattered light in ELS is much weaker and additionally includes intensity fluctuations. On the other hand, a high accuracy is required for ELS measurements. So far, an accuracy for $\tan \left(\Psi_{\mathrm{Q}}\right)$ data on the order $10^{-4}$ has been achieved (see refs $1-3$ or Figures 3 , 4, and 6 below). Since $\tan \left(\Psi_{\mathrm{Q}}\right)$ represents an amplitude ratio (see section 2 ), the accuracy corresponds to an intensity ratio of the two polarization modes of $10^{-8}$ !

An essential technique to achieve this accuracy is two-zone averaging, ${ }^{4,7}$ where two distinct pairs $\left(P_{0}, A_{0}\right)$ and $\left(P_{0}{ }^{\prime}, A_{0}{ }^{\prime}\right)$ of nulling angles are determined. A suitable averaging of these pairs eliminates most imperfections of the optical components as well as adjustment inaccuracies to first-order. A detection scheme often applied in reflection ellipsometry is based on separate scans on $P$ and $A$ in order to find $\left(P_{0}, A_{0}\right)$ or $\left(P_{0}{ }^{\prime}, A_{0}{ }^{\prime}\right)$. The transfer of such a procedure to ELS measurements was not successful. For the smallest value $\tan \left(\Psi_{\mathrm{Q}}\right)=10^{-4}$, the difference of the analyzer nulling angles $A_{0}=$ $\tan \left(\Psi_{\mathrm{Q}}\right)$ and $A_{0}{ }^{\prime}=-\tan \left(\Psi_{\mathrm{Q}}\right)$ of the two zones (see eq 7$)$ is only $0.01^{\circ}$. Also, a scan of $P$ to determine $\Delta$ shows only a tiny intensity modulation for such a situation, since $\tan \left(\Psi_{\mathrm{Q}}\right)$ limits (inequality 9 ) the amplitude factor $\tan \left(\Psi_{\mathrm{A}}\right)$. A high accuracy is achieved by a two-dimensional fit on the basis of eq 6 for a wide range of different $P$ and $A$ settings. To cover both pairs of nulling angles for typical values of $\tan \left(\Psi_{\mathrm{Q}}\right)$ and $\Delta$ close to $\theta_{\mathrm{B}}, A$ is varied from $162^{\circ}$ to $208^{\circ}$ in steps of $4^{\circ}$, whereas $P$ is scanned from $0^{\circ}$ to $180^{\circ}$ in steps of $22.5^{\circ}$. At each setting of $P$ and $A$, a scattering intensity measurement of typically $10 \mathrm{~s}$ duration is performed.

Equation 6 adapted to experimental conditions reads

$$
\begin{array}{r}
I(P, A)=I_{\mathrm{d}}+I_{V V}+I_{H H}+I_{\mathrm{s}}\left\{1+\cos ^{2}(\theta)\right\}- \\
\cos \left(2 A+2 \delta_{\mathrm{A}}\right)\left[I_{V V}-I_{H H}+I_{\mathrm{s}}\left\{1-\cos ^{2}(\theta)\right\}\right]+ \\
\sin \left(2 A+2 \delta_{\mathrm{A}}\right)\left[\left\{I_{V V}-I_{H H}\right\} \tan \left(2 \Psi_{\mathrm{Q}}\right) \sin (2 P-\Delta)\right]+ \\
\sin \left(2 A+2 \delta_{\mathrm{A}}\right) 2 I_{\mathrm{s}}|\cos (\theta)| \sin (2 P)
\end{array}
$$

The cross term $\left|\left\langle S_{1} * S_{2}\right\rangle\right|$ in eq 6 is eliminated using eq 5 . The normalized intensity and the elements $\left\langle\left|S_{1}\right|^{2}\right\rangle$ and $\left\langle\left|S_{2}\right|^{2}\right\rangle$ are replaced by the corresponding experimental countrates $I(P, A), I_{V V}$, and $I_{H H}$, respectively. There is no need to normalize, since eqs $3-5$ always involve a ratio of two countrates. As additional fixed experimental parameters, $I_{\mathrm{d}}$ denotes the detector dark signal and $I_{\mathrm{s}}$ the solvent's $V V$ scattering. The $H H$ scattering of the solvent is described by $I_{\mathrm{s}}$ $\cos ^{2}(\theta)$, and the phase shift of pure solvent is assumed to jump sharply from $0^{\circ}$ to $180^{\circ}$ exactly at $\theta=90^{\circ}$. Depolarized light scattering of the solvent is not considered. This is a good approximation for water, a common solvent of high contrast. The variable parameter $\delta_{\mathrm{A}}$ accounts for a deviation between the two zones. Although such a deviation does not affect the final results of $\tan \left(\Psi_{\mathrm{Q}}\right)$ and $\Delta$ to first order in $\delta_{\mathrm{A}}$ (two-zone averaging), it has to be considered in the fitting function for a suitable representation of the data. Formally, $\delta_{\mathrm{A}}$ represents an adjustment error of the analyzer. In the fit, $I_{\mathrm{d}}$ and $I_{\mathrm{s}}$ are kept at constant values, which are determined in separate measurements. The remaining five variable parameters are $I_{V V}, I_{H H}, \Psi_{\mathrm{Q}}, \Delta$, and $\delta_{\mathrm{A}}$. As further parameters of interest, $\tan \left(\Psi_{\mathrm{I}}\right)$, $\tan \left(\Psi_{\mathrm{A}}\right)$, and $\tan \left(\Psi_{\mathrm{Q}}\right)$ are calculated with the help of eqs 3-5, where $\left\langle\left. S_{1}\right|^{2}\right\rangle$ and $\left\langle\left. S_{2}\right|^{2}\right\rangle$ are replaced by $I_{V V}$ and $I_{H H}$. 

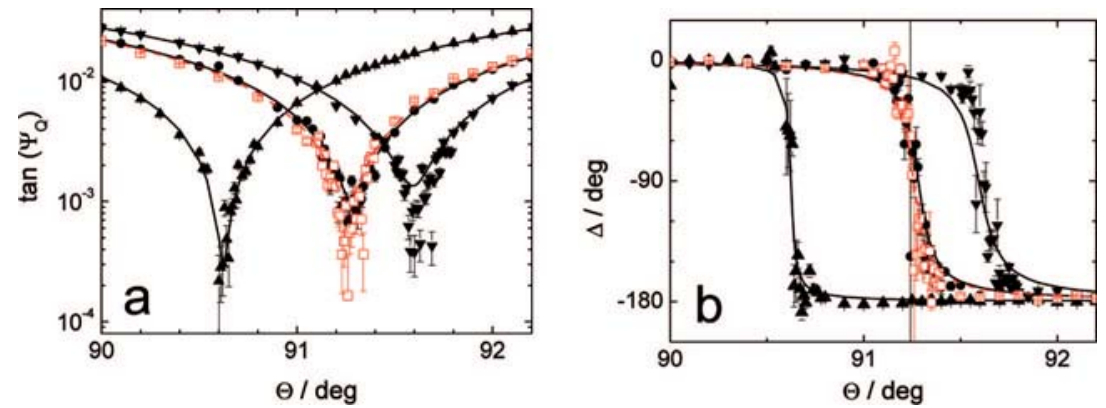

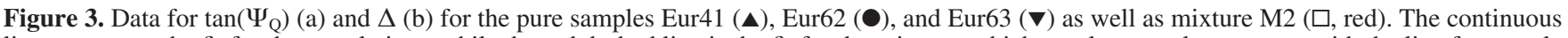
lines represent the fit for the pure latices, while the red dashed line is the fit for the mixture, which overlaps to a large extent with the line for sample Eur62.
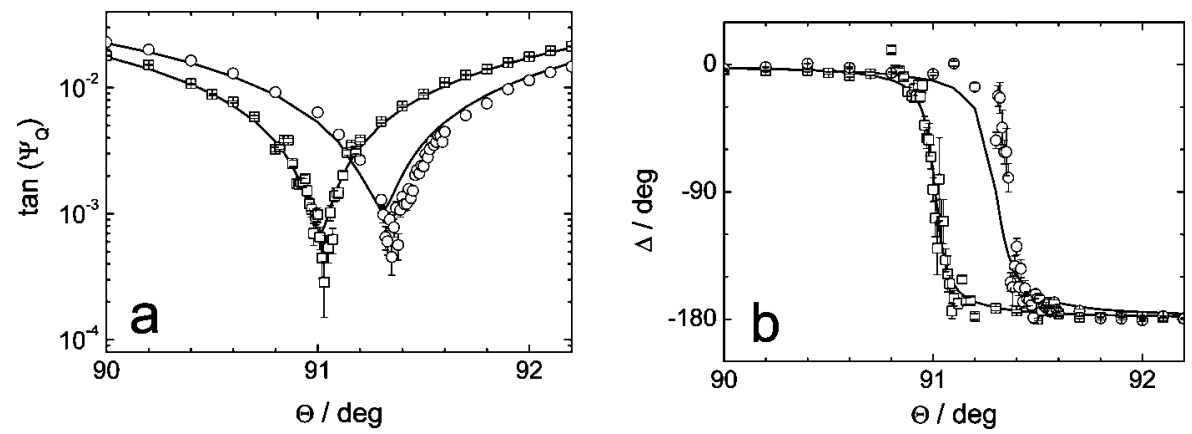

Figure 4. Data for $\tan \left(\Psi_{\mathrm{Q}}\right)$ (a) and $\Delta$ (b) for the mixtures M1 ( $\square$ ) and M3 (O). The continuous lines represent the fits.
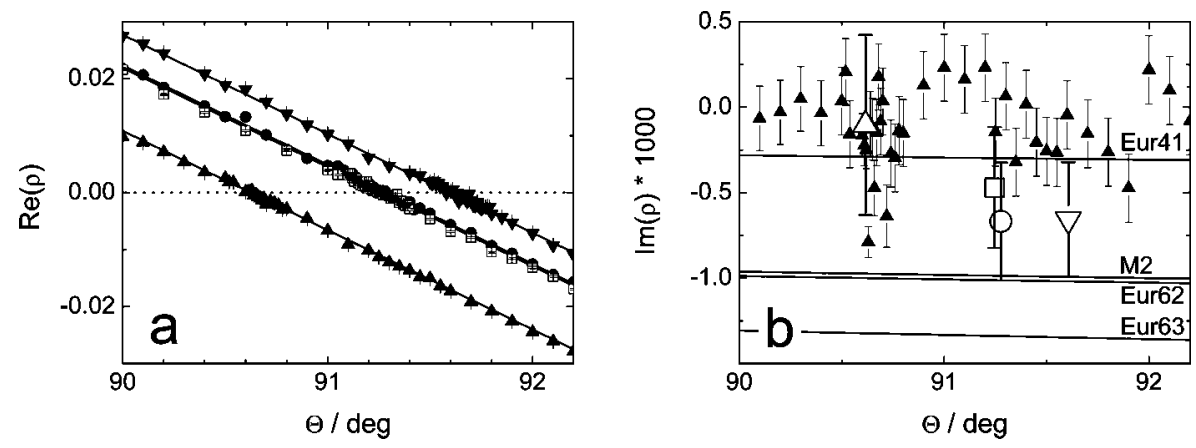

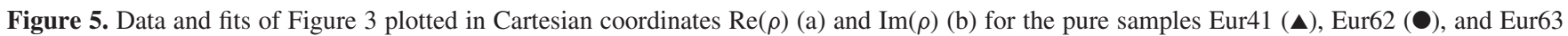
$(\boldsymbol{\nabla})$ and the mixture M2 ( $\square$ ). In part b, only data for Eur41 are included for clarity, while the open symbols indicate $\theta_{\mathrm{B}}$ and the averages and distribution widths of $\operatorname{Im}(\rho)$ data.

In a further treatment of the measured data, the results obtained for $\Delta$ have to be corrected for the birefringence of optical windows. This has been done by subtracting a constant offset of $8.7^{\circ} \pm 0.5^{\circ}$ (at $\lambda_{\mathrm{r}}$ ) or $11.9^{\circ} \pm 0.5^{\circ}$ (at $\lambda_{\mathrm{g}}$ ). ${ }^{1}$ The offsets were determined in measurements at small and large $\theta$, far away from $\theta_{\mathrm{B}}$. Finally, the sign of $\Delta$ for $\lambda_{\mathrm{g}}$ has to be flipped, since the retardation introduced by the compensator $\mathrm{C} 1$ is of opposite sign compared to the retardation for $\lambda_{\mathrm{r}}$ (see section 3.1).

The characteristics of the particles are afterward extracted from the $\tan \left(\Psi_{\mathrm{Q}}\right)$ vs $\theta$ and $\Delta$ vs $\theta$ curves by fitting a model based on Mie theory. The fitting procedure used was the "downhill simplex method" integrated into a simulated annealing algorithm slightly adapted from ref 8 . The errors were determined by the "bootstrap method" as described in ref 8 . A current version of the resulting program dplsianl.exe is available on the Internet. ${ }^{9}$

Whereas in reflection ellipsometry frequently high speed measurements are the target, the measurement scheme described here is optimized for high accuracy. The achieved high resolution in

(8) Press, W. H.; Teukolsky, S. A.; Vetterling, W. T.; Flannery, B. P. Numerical Recipes in C; Cambridge University Press: Cambridge, 1992.

(9) http://home.arcor.de/aerbe/en/prog/dplsianl.html

(10) Walz, R.; Bömer, B.; Heitz, W. Macromol. Chem. 1977, 178, 2527-2534. $\tan \left(\Psi_{\mathrm{Q}}\right)$ is visualized in logarithmic plots in section 4 . In reflection ellipsometry, a logarithmic representation of $\tan (\Psi)$ is not common.

3.3. Samples. PS particles of three different sizes (Eur41, Eur62, and Eur63) were synthesized by standard emulsion polymerization. The reaction mixture consisted of $10 \mathrm{~g}$ of styrene monomer and sodium perfluorooctanoate (Eur41, 0.1 g; Eur62, 0.04 g; Eur63, $0.03 \mathrm{~g}$ ) in $35 \mathrm{~g}$ of double-distilled water. PEGA200 is a symmetrical azo initiator that is prepared by esterification of $2,2^{\prime}$-azobisisobutyronitrile with poly(ethylene glycol) of a molecular weight of 200 $\mathrm{g} / \mathrm{mol}$ as described in ref 10 . The azo group decomposes at elevated temperatures and leads to the formation of carbon radicals with attached poly(ethylene glycol) units that can start polymerization. ${ }^{11}$ The mixture was heated to $80{ }^{\circ} \mathrm{C}$ under stirring and purging with nitrogen. After thermal equilibration, $0.673 \mathrm{~g}$ of PEGA200 dissolved in $5 \mathrm{~g}$ of double-distilled water was added to initiate the polymerization. After the polymerization was completed $(3 \mathrm{~h})$, coagulum was removed from the latex by filtration through a sintered glass frit. The obtained particles were diluted to a weight fraction of $2.6 \times$ $10^{-5}$ (Eur41) or $1 \times 10^{-5}$ (Eur62 and Eur63) with deionized water.

(11) Tauer, K.; Antonietti, M.; Rosengarten, L.; Müller, H. Macromol. Chem. Phys. 1998, 199, 897-908. 
Table 1. Weight Fractions $w_{\text {EUR41 }}, w_{\text {EUR62 }}$, and $w_{\text {EUR63 }}$ of Pure Latex Samples in Three Mixtures M1, M2, and M3 and the Calculated Polydispersity $M_{\mathrm{w}} / M_{\mathrm{n}}$

\begin{tabular}{llll}
\hline & M1 & M2 & M3 \\
\hline$w_{\text {EUR41 }} \times 10^{6}$ & 7.3 & 6.8 & 8.4 \\
$w_{\text {EUR62 }} \times 10^{6}$ & 8.8 & 4.0 & 0 \\
$w_{\text {EUR63 }} \times 10^{6}$ & 0 & 3.5 & 7.2 \\
$M_{\mathrm{w}} / M_{\mathrm{n}}$ & 1.16 & 1.19 & 1.22
\end{tabular}

Table 2. Particle Sizes of the Pure Samples Eur41, Eur62, and Eur63: Hydrodynamic Radius $\boldsymbol{R}_{\mathrm{h}}$, the Form Factor Radius $\boldsymbol{R}_{\mathrm{ff}}$, and Radius $\boldsymbol{R}_{\mathrm{e}}$ from ELS

\begin{tabular}{cccc}
\hline & Eur41 & Eur62 & Eur63 \\
\hline$R_{\mathrm{h}} / \mathrm{nm}$ & $66 \pm 2$ & $85 \pm 3$ & $97 \pm 3$ \\
$R_{\mathrm{ff}} / \mathrm{nm}$ & $65.8 \pm 0.4$ & $85.2 \pm 0.4$ & $94.3 \pm 0.3$ \\
$R_{\mathrm{e}} / \mathrm{nm}$ & $58.0 \pm 0.5$ & $78.3 \pm 0.5$ & $85.3 \pm 0.3$
\end{tabular}

The pure latex samples were initially characterized by static (SLS) and dynamic (DLS) light scattering. The same setup as described in section 3.1 has been used, where $\mathrm{C} 1$ and $\mathrm{C} 2$ were effectively deactivated by rotating them parallel to $\mathrm{P}$ and A, respectively. SLS data in $V V$ geometry was fit to the Mie scattering form factor of a monodisperse sphere ${ }^{12}$ to yield the radius $R_{\mathrm{ff}}$. For the particle sizes and the contrast ratio considered here, the Mie scattering value for $R_{\mathrm{ff}}$ is $1-2 \mathrm{~nm}$ smaller than the result from a Rayleigh-Debye form factor analysis. The connection to the radius of gyration $R_{\mathrm{g}}$ often used in SLS data evaluation is $R_{\mathrm{g}}{ }^{2}=3 / 5 R_{\mathrm{ff}}{ }^{2}$. For a comparison with $\tan \left(\Psi_{\mathrm{I}}\right)$ data from ELS, additional measurements in $H H$ geometry were performed.

The hydrodynamic radius $R_{\mathrm{h}}$ was determined by angular-dependent DLS measurements. Data for the apparent diffusion coefficient were extrapolated to the squared scattering vector $q^{2}=0$. The resulting diffusion coefficient was inserted into the Stokes-Einstein equation to calculate $R_{\mathrm{h}}{ }^{13}$

The refractive index increment of the particle dispersions has been measured using a ScanRef interferometric refractometer (Nanofilm, Göttingen, Germany) at $\lambda_{\mathrm{r}}$.

The characteristics of three mixtures, M1, M2, and M3, of the dispersions are shown in Table 1 . Beside the weight fractions $w_{\text {EUR } 41}$, $w_{\text {EUR62 }}$, and $w_{\text {EUR63 }}$ of the pure samples, the calculated polydispersity $M_{\mathrm{w}} / M_{\mathrm{n}}$ is listed. This ratio of weight-averaged molecular weight $M_{\mathrm{w}}$ and number-averaged molecular weight $M_{\mathrm{n}}$ is a measure commonly used in colloid and polymer science to quantify the polydispersity of a sample. Here, $M_{\mathrm{w}}$ and $M_{\mathrm{n}}$ refer to the entire colloidal particle. The calculation of $M_{\mathrm{w}} / M_{\mathrm{n}}$ is based on $R_{\mathrm{ff}}{ }^{3}$ (see below, Table 2) as a measure of the particles molecular mass. Although the differences in $M_{\mathrm{w}} / M_{\mathrm{n}}$ may appear small, there are clearly detectable effects in ELS.

\section{Results and Discussion}

4.1. Average Sizes of Particle Mixtures. For an experimental check of the predicted effect of polydispersity, ELS measurements from three latex particle samples and from model mixtures from these samples with a controlled polydispersity are compared.

The components of the model mixtures were characterized by SLS, DLS, and ELS. A Guinier plot of SLS data with fits to the Mie scattering form factor of a solid sphere is displayed in Figure 2.

An overview of the particle sizes of the pure samples determined by SLS and DLS is provided in Table 2.

SLS data for the mixtures are also included in Figure 2. The Mie scattering form factor of a homogeneous sphere enables a suitable fit also for the mixtures. The experimental window covers only the Guinier range, where the scattering data show an almost linear behavior in the Guinier plot. Details of the particle

(12) Kerker, M. The Scattering of Light and Other Electromagnetic Radiation; Academic Press: New York, 1969.

(13) Berne, B. J.; Pecora, R. Dynamic Light Scattering; Dover: Mineola, 2000.
Table 3. Results for $\boldsymbol{R}_{\mathrm{e}}$ from ELS, $\boldsymbol{R}_{\mathrm{ff}}$ from SLS, and $\boldsymbol{R}_{\mathrm{h}}$ from DLS for the Different Mixtures

\begin{tabular}{lccc}
\hline & M1 & M2 & M3 \\
\hline$R_{\mathrm{h}} / \mathrm{nm}$ & $81 \pm 3$ & $84 \pm 3$ & $91 \pm 4$ \\
$R_{\mathrm{ff}} / \mathrm{nm}$ & $79.0 \pm 0.4$ & $83.1 \pm 0.5$ & $83.9 \pm 0.4$ \\
$R_{\mathrm{e}} / \mathrm{nm}$ & $71.2 \pm 0.5$ & $77.8 \pm 0.5$ & $79.0 \pm 0.1$
\end{tabular}

morphology and the polydispersity are not resolved. The systematic upturn at low $q$ for M2 might indicate some aggregation. Fitting results for $R_{\mathrm{ff}}$ and $R_{\mathrm{h}}$ from SLS and DLS, respectively, are listed in Table 3 . The results agree fairly well with calculated values based on the mixture parameters given in Tables 1 and $2 .{ }^{13}$ Generally, $R_{\mathrm{ff}}>R_{\mathrm{h}}$ is expected for polydisperse samples. Here, however, the data quality is not sufficient for a confirmation. The experimental noise exceeds the effect, which is small for the mixtures of limited polydispersity.

ELS data for the pure samples are displayed in Figure 3. The characteristic right shift of $\theta_{B}$ for increasing particle size is visible in this overview.

The data were fit to a model of a homogeneous sphere of radius $R_{\mathrm{e}}$ according to Mie theory. This corresponds to the equation given in ref 1 without any layers. Since the stabilizing agent perfluorooctanic acid has a short chain length and is almost isorefractive to water, the interfacial profile of the particles is well-approximated by a step function with no interface layers present. The refractive index of the particle's core was fixed at the known bulk index $n_{\mathrm{PS}}=1.59$ of PS. ${ }^{14}$ The only free parameter in these fits is $R_{\mathrm{e}}$. The fits are also displayed in Figures 3 (M2) and 4 (M1). The results for $R_{\mathrm{e}}$ are included in Table 2. Although $R_{\mathrm{e}}$ shows the right trend, the values are characteristically lower than $R_{\mathrm{ff}}$ and $R_{\mathrm{h}}$. The deviation is discussed in detail in section 4.5.

Figure 3 also contains the measured data of mixture M2. The curves for $\tan \left(\Psi_{\mathrm{Q}}\right)$ and $\Delta$ of the mixture agree quite well with the curves obtained from the pure component Eur62. It is possible to use a model of a monodisperse sample to fit $\tan \left(\Psi_{\mathrm{Q}}\right)$ and $\Delta$ of the mixtures. The results are also included in Table 3. Again the change in size of $R_{\mathrm{e}}$ parallels the increasing values of $R_{\mathrm{ff}}$ and $R_{\mathrm{h}}$. However, as for the pure samples, $R_{\mathrm{e}}$ is characteristically lower than the reference values (see Table 2).

For the experiments reported here, only one wavelength of light was available. In this situation, the effects of particle size and refractive index are correlated, since they affect the location of $\theta_{\mathrm{B}}$ in a similar manner. For particles as small as considered here, a higher refractive index and a larger particle radius shift $\theta_{\mathrm{B}}$ to larger values. The observed too low value for $R_{\mathrm{e}}$ could therefore result from a high particle refractive index in the fit. As a cross check, an alternate fit with $R_{\mathrm{e}}=R_{\mathrm{ff}}$ fixed and varying particle refractive index was performed. For such an interpretation, a particle refractive index in the range $1.53-1.55$ would be required, significantly lower than the PS bulk index. To rule out this possibility, the refractive index of particles was estimated from measurements of the refractive index increment $\mathrm{d} n / \mathrm{d} c$ and the density $\rho_{\mathrm{PS}}$ of the particles. In the simplest manner, the refractive index $n_{\mathrm{P}}$ of the particle is estimated as $n_{\mathrm{P}}=n_{\mathrm{H}_{2} \mathrm{O}}+$ $\mathrm{d} n / \mathrm{d} c \rho_{\mathrm{PS}}$, where $n_{\mathrm{H}_{2} \mathrm{O}}$ is the refractive index of water. A more accurate method is based on the theoretical work of Zimm and Dandliker. ${ }^{16}$ The two methods yield $n_{\mathrm{P}}$ between 1.59 and 1.63 , which is in good agreement with the bulk index $n_{\mathrm{PS}}=1.59$ of $\mathrm{PS}^{14}$ used in the fits.

(14) Schrader, D. In Polymer Handbook, 4th ed.; Brandrup, J.; Immergut, E. H.; Grulke, E. A., Eds.; Wiley: Hoboken, 1999; pp, V/91-V/96.

(15) Schmidt, M. In Dynamic Light Scattering; Brown, W., Ed.; Clarendon: Oxford, 1993; pp 374-439.

(16) Zimm, B. H.; Dandliker, W. B. J. Phys. Chem. 1954, 58, 644-648. 


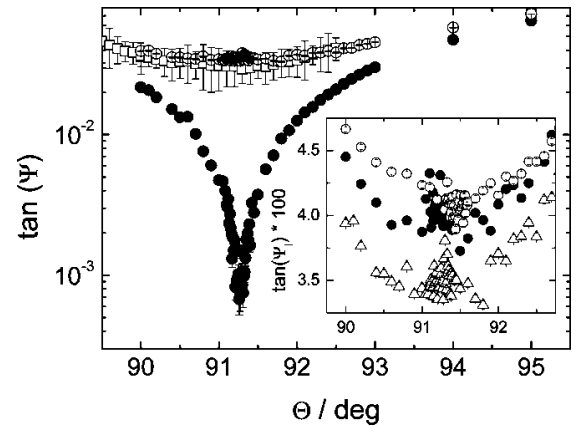

Figure 6. Comparison of $\tan \left(\Psi_{\mathrm{I}}\right)(\bigcirc)$ and $\tan \left(\Psi_{\mathrm{Q}}\right)(\bullet)$ for sample Eur62. Also shown is $\tan \left(\Psi_{\mathrm{I}}\right)$ calculated from SLS data $(\square)$. Inset: $\tan \left(\Psi_{\mathrm{I}}\right)$ for Eur62 $(\Delta)$, M2 $(\bullet)$, and M3 $(O)$.

The low $R_{\mathrm{e}}$ values can be attributed to radial birefringence of the particles, as discussed in section 4.5. $R_{\mathrm{e}}$ for particles with deviation from the isotropic spherical shape can be considered as an effective quantity. It can be understood as the radius of an isotropic homogeneous sphere of preset refractive index, which produces for given $\lambda$ the same location of the Brewster angle than the actual particle. Such a perception could be used as an analytic handle for ELS measurements on nonspherical particles. In the present work, the deviation is caused by radial birefringence (see section 4.5). Thus, a characteristic ratio $R_{\mathrm{e}} / R_{\mathrm{h}}$ or $R_{\mathrm{e}} / R_{\mathrm{ff}}$ could be used to classify the internal anisotropy, similar to the description of particle shape anisotropy by the charactersitic ratio $R_{\mathrm{g}} / R_{\mathrm{h}}$ (see ref 15).

4.2. Monodisperse Particles Representation. From the point of view of classical scattering, the similarity of the data (Figure 3) between the pure component Eur62 and the mixture M2 seems surprising. The details of the structured appearance of the $\tan \left(\Psi_{\mathrm{Q}}\right)$ and $\Delta$ data are well reproduced and an effect of polydispersity seems completely absent. A simpler shape is obtained in a representation of $\rho$ in Cartesian coordinates $(\operatorname{Re}(\rho), \operatorname{Im}(\rho))$ instead of the polar coordinates $(\tan (\Psi), \Delta)$ (see eq 1$)$, as depicted in Figure 5.

The $\operatorname{Re}(\rho)$ data for the different samples follow straight lines of equal slopes. The essential information is the root of the straight line, which indicates $\theta_{\mathrm{B}}$. For $\theta=\theta_{\mathrm{B}}, \rho$ becomes purely imaginary, $|\rho|=\tan (\Psi)$ is at minimum, and $\Delta= \pm 90^{\circ}$ marks the inflection point of the transition. Data for $\operatorname{Im}(\rho)$ are essentially constant for the small particle size considered here. There is a small slope in the fitting lines of $\operatorname{Im}(\rho)$, which, however, depends sensitively on the correction for window birefringence applied within the data treatment (see section 3.2). Within the experimental error, only the level of $\operatorname{Im}(\rho)$ can be extracted from the data. It determines the minimum value of $\tan \left(\Psi_{\mathrm{Q}}\right)$ and the steepness of change in $\Delta$. The distinction of the two possible transitions of $\Delta, 0 \rightarrow 180^{\circ}$ or $0 \rightarrow-180^{\circ}$, depends on the sign of $\operatorname{Im}(\rho)$. ELS data at one wavelength for particles as small as considered here can thus be reduced to two quantities, $\theta_{\mathrm{B}}$ and $\operatorname{Im}(\rho)$. Such a reduction is also possible for the mixtures. In Figure 5, the data for mixture M2 show the same slope in $\operatorname{Re}(\rho)$ and also a constant behavior of $\operatorname{Im}(\rho)$. A model of a monodisperse particle ensemble thus recovers the main features of $\tan \left(\Psi_{\mathrm{Q}}\right)$ and $\Delta$ data of a polydisperse sample at a single wavelength. For the employed one parameter fit, the particle radius is mainly determined by $\theta_{\mathrm{B}}$, while $\operatorname{Im}(\rho)$ is less well recovered. Generally, the fit produces too low $\operatorname{Im}(\rho)$ values.

4.3. Averaging in ELS. Because the ELS results here can be represented by monodisperse spheres, the question arises what kind of averaging takes place over the particle size. The presented data on mixtures can be used to address that question on an
Table 4. Comparison of the Experimental Results of $\boldsymbol{R}_{\mathrm{e}}$ for the Mixtures M1, M2, and M3 with Different Averages of Sizes from the Pure Components

\begin{tabular}{lccc}
\hline & M1 & M2 & M3 \\
\hline$R_{\mathrm{e}} / \mathrm{nm}(\exp )$ & $71.2 \pm 0.5$ & $77.8 \pm 0.5$ & $79.0 \pm 0.1$ \\
$\left(R_{\mathrm{e}}{ }^{-1}\right)_{z}^{-1 / n m}$ & $71.4 \pm 0.4$ & $73.9 \pm 0.3$ & $75.2 \pm 0.3$ \\
$\left(R_{\mathrm{e}}\right)_{z} / \mathrm{nm}$ & $72.7 \pm 0.4$ & $75.7 \pm 0.3$ & $77.6 \pm 0.3$ \\
$\left(R_{\mathrm{e}}{ }^{1 / 2}\right)_{z}^{1 / \mathrm{nm}}$ & $73.3 \pm 0.8$ & $76.5 \pm 0.5$ & $78.5 \pm 0.6$ \\
$\left(R_{\mathrm{e}}{ }^{3}\right)^{1 / 3} / \mathrm{nm}$ & $73.8 \pm 1.3$ & $77.3 \pm 0.8$ & $79.4 \pm 0.4$
\end{tabular}

empirical basis. The different averages are calculated from a mass distribution with fractions $w_{i}$ of well-defined molecular weight $M_{i}(i \in\{1, \ldots, N\}) .{ }^{17}$ The $v$ th moment of a quantity $x$ with values $x_{i}$ for the different fractions reads

$$
\left(x^{\nu}\right)_{\mu}=\frac{\sum_{i} w_{i} R_{\mathrm{ffi} i}{ }^{\mu} x_{i}^{\nu}}{\sum_{j} w_{j} R_{\mathrm{ff} j}{ }^{\mu}}
$$

Here, $\mu$ describes the type of distribution; for $\mu=-3, \mu=$ 0 , and $\mu=3$ the result is the moment for a number distribution, a weight distribution, and an intensity weighted distribution, respectively. The relation $M_{i} \sim R_{\mathrm{ff}}{ }^{3}$, valid for spherical particles, was inserted for the transformations, neglecting the polydispersity of the particle density.

The effects of size averaging are known for SLS and DLS, where low $q^{2}$ expansions yield simple expressions for the averages. ${ }^{13}$ Since both techniques detect particles via the scattered light, they provide intensity-weighted averages, also called $z$-averages. While DLS determines the $z$-average of the diffusion constant corresponding to a $z$-averaging of $R_{\mathrm{h}}{ }^{-1}$, SLS yields the $z$-average of $R_{\mathrm{ff}}{ }^{2}$. Due to this different averaging, the characteristic ratio $R_{\mathrm{ff}} / R_{\mathrm{h}}$ exceeds 1 for polydisperse spherical particles.

In the case of ELS, there is no simple and generally valid formula for the type of size averaging of a polydisperse sample. The location of $\theta_{\mathrm{B}}$ calculated as a minimum of a quotient of two infinite Mie series has much more complicated analytic properties than the low $q^{2}$ expansions of SLS and DLS. For the small particle sizes investigated here, however, the fitting of $\tan \left(\Psi_{\mathrm{Q}}\right)$ and $\Delta$ from a polydisperse sample with a simple monodisperse particle model was found to work fine. With the mixing ratios (Table 1) and the sizes of the pure components (Table 2), different moments $(v=-1,1,2,3$ in eq 11) of the radius distributions weight by the respective arguments number (n), weight (w), and $z$ were calculated, neglecting the small polydispersity of the pure components. All number averages and weight averages are significantly lower than the experimental results (Table 3). This finding is not unexpected, since the intensity-weighted detection applies to ELS measurements as to other light-scattering methods. The different moments with respect to $z$ are listed in Table 4 . The values $-1,2$, and 3 for $v$ result in an averaging similar to the averaging of $R_{\mathrm{h}}, R_{\mathrm{ff}}$, and the particle molecular weight, respectively. For mixtures M2 and M3, $z$-averaging of the third moment describes the ELS results best, while for M1, the negative first moment is closest to the experimental result.

When interpreting the experimental results, one should always caution oneself to notice that the differences observed here are within a few nanometers only. In addition, the experiments do also suffer from the superimposed effects of particle birefringence (section 4.5), the neglected effect of density polydispersity and the errors of $R_{\mathrm{ff}}$ entering into the results.

Since the experimental results turn out to be inconclusive, numerical simulations of the averaging have been performed.

(17) Elias, H. G. Makromoleküle: 2. Physikalische Strukturen und Eigenschaften; Wiley-VCH: Weinheim, 2001. 
For these simulations, the complex scattering amplitudes $S_{1}$ and $S_{2}$ of the individual particles where computed, and then averages according to eqs $2-5$ were evaluated according to an imposed composition. The resulting curves for $\Delta$ and $\tan \left(\Psi_{\mathrm{Q}}\right)$ where subsequently subjected to the same fit as the experimental results. As a result of the fits, average particle radii were obtained. Comparing these radii to the different moments of the $z-$ distribution calculated from the composition in most of the calculated examples gives results between $\mu=-1$ and 1 , similar to that experimentally observed for M1. However, examples with a small fraction of larger particles give results close to $\mu$ $=2$. So far, in the numerical experiments we never observed the $v=3$ found in the experiments. While the averaging in ELS clearly involves intensity weighting, the exact moment to use depends on the details of the size distribution.

4.4. Effect of Polydispersity on ELS. Another quantity obtained from ELS measurements is $\tan \left(\Psi_{\mathrm{I}}\right)$. A comparison of $\tan \left(\Psi_{\mathrm{I}}\right)$ and $\tan \left(\Psi_{\mathrm{Q}}\right)$ for the pure latex sample Eur62 is shown in Figure 6.

Data for $\tan \left(\Psi_{\mathrm{I}}\right)$ can also be determined from two SLS measurements in $V V$ and $H H$ geometry (see section 2). The curves for $\tan \left(\Psi_{\mathrm{I}}\right)$ derived by ELS and by SLS for one sample under the same conditions agree quite well, as exemplified in Figure 6 for Eur62. The large errors in the SLS-derived data are due to the low scattering intensity in $H H$ geometry around $\theta_{\mathrm{B}}$. As expected from eq $9, \tan \left(\Psi_{\mathrm{I}}\right)$ exceeds $\tan \left(\Psi_{\mathrm{Q}}\right)$. Especially around $\theta_{\mathrm{B}}$, the deviations become clearly visible in the logarithmic representation of Figure 6. Since this major difference is already observed for the unmixed samples of low polydispersity, it can be attributed to the effect of the beam profile of the incident light and other nonidealities. 4

The inset in Figure 6 displays $\tan \left(\Psi_{\mathrm{I}}\right)$ measurements of one pure latex and two different mixtures, showing an increase in $\tan \left(\Psi_{\mathrm{I}}\right)$ with increasing polydispersity. The change of $\tan \left(\Psi_{\mathrm{I}}\right)$ occurs on a linear scale and it is possible to estimate the polydispersity from $\tan \left(\Psi_{\mathrm{I}}\right)$ data. However, for an exact determination, the extent of incoherent scattering due to the beam profile and other sources has to be known, e.g. by a calibration measurement on samples with negligible polydispersity.

4.5. Particle Birefringence. Compared to $R_{\mathrm{ff}}$ and $R_{\mathrm{h}}$, the values obtained for $R_{\mathrm{e}}$ are systematically too low (section 4.1). Beside the particle radius and refractive index, also an internal anisotropy can affect the location of $\theta_{\mathrm{B}}$. In a recent work, we found a significant shift of $\theta_{\mathrm{B}}$ for vesicles with a radially birefringent shell. ${ }^{3}$ Such radial birefringence does not lead to significant depolarized $(V H)$ scattering $^{3}$ and is therefore difficult to detect in classical scattering methods. ${ }^{18}$ For the particles investigated here, the $V H$ scattering intensity was indeed very low $\left[I_{V H} / I_{V V}\right.$ $\left.=\mathrm{O}\left(10^{-4}\right)\right]$.

A possible effect of anisotropy was cross-checked with fits of a model of a birefringent sphere. A core-shell model particle with a minimum sized isotropic core (radius $1 \mathrm{~nm}$ ) and a birefringent shell with an average refractive index $n_{\mathrm{PS}}=1.59$ and a fixed total radius equal to $R_{\mathrm{ff}}$ was used. The only free parameter was the birefringence $\Delta n=n_{\mathrm{n}}-n_{\mathrm{t}}$. Here, $n_{\mathrm{t}}^{2}$ and $n_{\mathrm{n}}{ }^{2}$ are the dielectric constants for light with the electric field vector tangential [transversal electric (TE) mode] and normal [transversal magnetic (TM) mode] to the local interface, respectively. For the three pure latices, $\Delta n$ values between 0.03 and 0.04 were obtained from the fits. An example for Eur63 is shown in Figure 7. While both models recover $\theta_{\mathrm{B}}$ equally well, the model of the

(18) Desbordes, M.; Meeten, G. H.; Navard, P. J. Polym. Sci. Polym. Phys. Ed 1989, 27, 2037-2043.

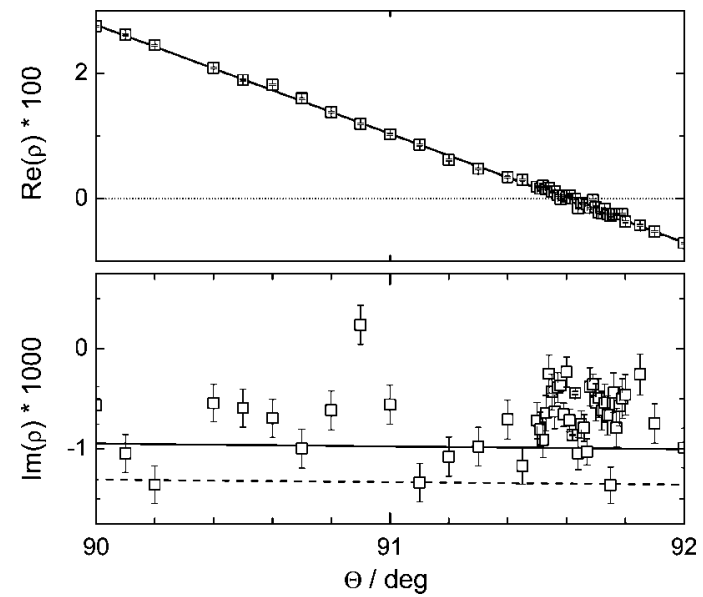

Figure 7. $\operatorname{Re}(\rho)$ (top) and $\operatorname{Im}(\rho)$ (bottom) for $\operatorname{Eur63}(\square)$ with fits of an isotropic sphere $(--)$ and a birefringent sphere $(-)$ (see the text). For $\operatorname{Re}(\rho)$, the fits overlap.

birefringent sphere significantly improves the quality of the fit for $\operatorname{Im}(\rho)$.

An alternate fit of the data with only a thin anisotropic layer of a few angstroms comparable to the width $d_{\sigma}$ of the interfacial profile leads to unphysically high values for $\Delta n$. A reasonable model requires radial symmetric anisotropy within the whole particle.

An interpretation of the optical anisotropy in the colloids is based on the linear stress optical rule for polymers ${ }^{19}$

$$
\Delta n=n_{\perp}-n_{z}=C\left(\Sigma_{z z}-\Sigma_{\perp}\right) .
$$

In eq 12 , a cylindrical stress geometry with symmetry axis along the stretching direction $z$ is described. The birefringence $\Delta n$ is connected via the stress optical coefficient $C$ to the difference between the stress $\Sigma_{z z}$ along the $z$ axis and the stress $\Sigma_{\perp}$ perpendicular to the $z$ axis. The electric field vector of light propagating in the $z$ direction is perpendicular to $z$, and therefore, its speed is determined by $n_{\perp}$. On the other hand, $n_{z}$ describes the speed of light with electric field parallel to the $z$-axis with a propagation perpendicular to $z$.

In spherical particles with radial anisotropy, the stresses for the cylindrically symmetric problem need to be replaced by those of radial symmetry, i.e., the radial stress $\Sigma_{\mathrm{r}}$ and the tangential stress $\Sigma_{\mathrm{t}}$. Here, an anisotropic stress difference can be caused by the interface tension $\sigma$ between water and PS. The description of an interface by $\sigma$, however, assumes a dividing surface of two phases of thickness zero. In order to transform $\sigma$ (unit of force per length) to a stress (unit of force per area), an additional length scale is needed. The scale describes the extension of an interface layer that supports the stress induced by the interface tension. The idea of a homogeneous radial anisotropic particle, on the other hand, relies on the assumption that the interior parts were at the interface during particle growth and therefore they were also affected by the interface tension effect. Note, that a smaller size of the interfacial layer translates to higher local stress and therefore higher birefringence.

A first assumption for the depth on which $\sigma$ affects the polymer configuration is $d_{\sigma}$. It turns out, however, that such an approach leads to a too high $\Delta n$.

A different length scale originates from polymer physics. Only for polymers with a molecular weight above the entanglement molecular weight $M_{\mathrm{c}}$, a rubber plateau is observed in the rheological properties of a polymer melt. ${ }^{19}$ The melt of such a

(19) Strobl, G. The Physics of Polymers; Springer: Berlin, 1996. 
polymer behaves as a rubber at intermediate oscillation frequencies and can support stress. For a polymer melt like the particle core, excluded volume interactions between chain segments are screened and the mean squared end-to-end distance $\left\langle r_{0 \mathrm{f}}^{2}\right\rangle$ of a coil is unperturbed and scales with its molecular weight $M_{\mathrm{w}} \cdot{ }^{19}$ The ratio $\left(\left\langle r_{0 \mathrm{f}}{ }^{2}\right\rangle / M_{\mathrm{w}}\right)^{1 / 2}$ is a specific constant for a polymer. A characteristic length scale $l_{\mathrm{c}}$ results as

$$
l_{\mathrm{c}}=\sqrt{M_{\mathrm{c}} \frac{\left\langle{\left.r_{0 \mathrm{f}}{ }^{2}\right\rangle}_{M_{\mathrm{w}}}\right.}{M^{2}}}
$$

For PS, $M_{\mathrm{c}}=19100 \mathrm{~g} / \mathrm{mol}^{14}$ and $\left(\left\langle r_{0 \mathrm{f}}{ }^{2}\right\rangle / \mathrm{M}_{w}\right)^{1 / 2}=0.0302 \mathrm{~nm} /$ $(\mathrm{g} / \mathrm{mol})^{1 / 2} ;{ }^{22}$ thus, $l_{\mathrm{c}} \simeq 4 \mathrm{~nm}$. Roughly, $l_{\mathrm{c}}$ can be addressed as the extension of an entanglement. This extension is the relevant depth at which the effect of the interface tension affects the polymer conformation. The difference between $\Sigma_{\mathrm{r}}$ and $\Sigma_{\mathrm{t}}$ at the particle interface reads

$$
\Sigma_{\mathrm{r}}-\Sigma_{\mathrm{t}}=-\frac{\sigma}{l_{\mathrm{c}}}
$$

At the interface, $\sigma$ creates a biaxially symmetric stress, which can be identified with a uniaxial compression. Therefore, the stress difference in eq 14 is negative. With a typical interface tension $\sigma \approx 10 \mathrm{mN} / \mathrm{m}$ of a PS latex/water interface ${ }^{20}$ and $C=$ $-4.6 \times 10^{-9} \mathrm{~Pa}^{-1}$ for $\mathrm{PS},{ }^{21}$ the particle birefringence results from eqs 12 and 14 as $\Delta n \simeq 0.01$. Compared to the experimental result, this value is of the right order of magnitude and therefore confirms $l_{\mathrm{c}}$ as the relevant length scale.

From a rheological point of view, the occurrence of $l_{\mathrm{c}}$ implies that there is still sufficient mobility in the interfacial layer. A relaxation to the rubber plateau takes place on a time scale compatible with the particle growth rate. In contrast, the glass modulus of a completely frozen system is independent of $M_{\mathrm{w}}$. No effect of $M_{\mathrm{c}}$ and therefore $l_{\mathrm{c}}$ is expected for a glassy frozen interface layer.

During emulsion polymerization, the colloid particles are the main locus where the polymerization reaction takes place. The particles swell with monomer from the monomer emulsion droplets. The latter do not participate actively in the polymerization. ${ }^{23}$ The monomer enters the particles from the water side. Hence, it is not equally distributed in the particle volume but rather it builds up a gradient toward the water phase. This was experimentally proven by X-ray scattering experiments of latex particle swelling. ${ }^{24}$ These results point for the methyl methacrylate emulsion polymerization to an outer shell of approximately 2 $\mathrm{nm}$ thickness in which the swelling agent is enriched.

\section{Conclusions}

For the recently established ELS, this work shows that the way in which polydispersity affects the measurements is

(20) Pieranski, P. Phys. Rev. Lett. 1980, 45, 569-572.

(21) Luap, C.; Müller, C.; Schweizer, Th.; Venerus, D. C. Rheol. Acta 2005, 45, 83-91.

(22) Kurata, M.; Tsunashima, Y. In Polymer Handbook, 4th ed.; Brandrup, J., Immergut, E. H., Grulke, E. A., Eds.; Wiley: Hoboken, 1999; pp, VII/1 - VII/ 83.

(23) Tauer, K.; Hernandez, H.; Kozempel, S.; Lazareva, O.; Nazaran, P. Colloid Polym. Sci. 2008, 286, 499-515.

(24) Boltze, J.; Ballauff, M. Macromolecules 1996, 28, 7429-7433. substantially different from classical scattering methods. The reason is that an extraction of the coherent scattering contribution in ELS is possible with high accuracy. The separation relies on a "lock-in"-like detection scheme, where the sinusoidal intensity variation with changing polarizer and analyzer angles allows the detection of coherent scattering, even when the incoherent scattering is largely dominant, e.g., close to the Brewster angle $\theta_{\mathrm{B}}$. In this regime, the classical scattering methods are dominated by incoherent scattering. The described two-dimensional scan of $P$ and $A$ in combination with the fit of eq 10 is the key to the achieved high accuracy.

From an ELS experiment, three quantities are extracted: $\tan \left(\Psi_{\mathrm{Q}}\right), \tan \left(\Psi_{\mathrm{I}}\right)$ and $\Delta$. Two of these, $\tan \left(\Psi_{\mathrm{Q}}\right)$ and $\Delta$, contain only coherent scattering contributions, hence representing monodisperse particles with averaged properties. For the small particles considered here, the radius of this "average particle" is welldescribed by a z-average of the radius of the mixture's components. The exact moment of the particle size distribution that is determined depends on the details of the sample composition. There is no simple integer, generally valid moment of the intensity-weighed size distribution that can be assigned to ELS results. The third experimental quantity $\tan \left(\Psi_{\mathrm{I}}\right)$-governed by the total scattering intensity and also accessible from static light scattering measurements - is affected by polydispersity. Its minimum is getting less and less pronounced with increasing polydispersity. A quantitative extraction of the polydispersity requires a detailed calibration of all effects, leading to a decrease in the light's degree of polarization. This includes but is not limited to incoherent scattering processes.

As shown previously, ${ }^{3}$ ELS is sensitive to anisotropy of particles. This sensitivity has been used here to determine the stress birefringence of the employed poly(styrene) particles. Such kind of latex particles were usually regarded as being isotropic. ${ }^{18}$ The magnitude of the birefringence was rationalized as a stress optical effect induced by the interface tension acting on the particle/water interface during the production of the particles.

For the main application of ELS, namely, the characterization of layers on colloidal particles, the findings presented here verify that for low polydispersity, the polydispersity does not enter the measurement process. For future applications, this is as important as the sensitivity to particle anisotropy. In order to make ELS applicable to a wider range of systems, the effects occurring for larger particles that have a form factor minimum in the accessible angular range need to be understood, as do the results for nonspherical particles. It is expected that once the measurements for monodisperse particles are understood, the same principles as proven here for small particles will also apply to polydisperse, large particles and nonspherical particles.

Acknowledgment. Financial support of the Max Planck Society is gratefully acknowledged. A.E. would like to acknowledge support of Chiafu Chou, Institute of Physics, Academia Sinica. R.S. thanks Peter Schurtenberger for support. 